University of Wollongong

Research Online

Faculty of Informatics - Papers (Archive)

Faculty of Engineering and Information

Sciences

October 2003

\title{
Performance Measurement of Watermark Embedding Patterns
}

\author{
R. Scealy \\ University of Wollongong \\ R. Safavi-Naini \\ University of Wollongong, rei@uow.edu.au \\ N. P. Sheppard \\ University of Wollongong,nps@uow.edu.au
}

Follow this and additional works at: https://ro.uow.edu.au/infopapers

Part of the Physical Sciences and Mathematics Commons

\section{Recommended Citation}

Scealy, R.; Safavi-Naini, R.; and Sheppard, N. P.: Performance Measurement of Watermark Embedding Patterns 2003.

https://ro.uow.edu.au/infopapers/415

Research Online is the open access institutional repository for the University of Wollongong. For further information contact the UOW Library: research-pubs@uow.edu.au 


\title{
Performance Measurement of Watermark Embedding Patterns
}

\author{
Abstract \\ Segmented watermarking is an approach to multiple watermarking that can be used to acknowledge the \\ contribution of multiple authors or to embed a fingerprint code-word, but is susceptible to cropping \\ attacks that discard portions of the watermarked media. Careful selection of the watermark embedding \\ pattern can increase the resilence of a segmented watermark in the presence of cropping attacks. In this \\ paper, we consider performance measures for embedding patterns and compare the performance of \\ several proposed embedding patterns using these measures. \\ Keywords \\ digital watermarking, fingerprinting \\ Disciplines \\ Physical Sciences and Mathematics \\ Publication Details \\ This paper was originally published as: Scealy, R, Safavi-Naini, R \& Sheppard, NP, Performance \\ Measurement of Watermark Embedding Patterns, Second International Workshop on Digital \\ Watermarking 2003 (IWDW 2003), Seoul, Korea, October 20-22 2003, 77-85.
}




\title{
Performance Measurement of Watermark Embedding Patterns
}

\author{
Robert Scealy, Reihaneh Safavi-Naini, and Nicholas Paul Sheppard \\ School of Information Technology and Computer Science \\ The University of Wollongong NSW 2522 \\ Australia \\ $\{$ rcs07, rei, nps $\}$ @uow.edu.au
}

\begin{abstract}
Segmented watermarking is an approach to multiple watermarking that can be used to acknowledge the contribution of multiple authors or to embed a fingerprint code word, but is susceptible to cropping attacks that discard portions of the watermarked media. Careful selection of the watermark embedding pattern can increase the resilience of a segmented watermark in the presence of cropping attacks. In this paper, we consider performance measures for embedding patterns and compare the performance of several proposed embedding patterns using these measures.
\end{abstract}

\section{Introduction}

In a segmented watermarking scheme [6], the object to be watermarked is divided into a series of segments, and each segment is watermarked independently. In this way, the ownership of multiple owners can be represented by embedding each of the owners' watermarks into a different segment, or a fingerprint code word can be encoded by embedding one code letter into each segment.

This method is obviously subject to a cropping attack, in which an attacker may discard some of the segments of no interest to him or her, possibly eliminating one or more watermarks or code letters from the object. Since, in general, there may be more segments than watermarks or code letters, and each watermark or code letter may be embedded multiple times, we can expect the segmented watermark to have at least some resilience against this kind of attack.

The embedding pattern chosen for mapping watermarks or letters to segments will affect the resilience of the overall watermark to these kinds of attacks. A good embedding pattern should allocate watermarks and letters to segments in such a way as to minimise the possibility of a watermark being eliminated or a code word being lost.

In applications where each segment represents a fingerprint code letter, we consider the case where the mark in each segment encodes both the letter itself and its position in the code word, that is, it encodes $\left(i, c_{i}\right)$ for position $i$ and letter $c_{i}$. In another approach, the letter's position in the code word might be implied only by the location of the segment in the original object. However, this 
location information is likely to be lost in any significant cropping attack. The measure of resilience of the former kind of encoding is the same as in the case where each segment represents an owner: after a cropping attack, we would like to have at least one segment corresponding to each $i \in\{1, \ldots, m\}$ for a set of $m$ owners or code words of length $m$.

In this paper, we consider segmented watermarking of still images by dividing them into rectangular blocks, and an attacker who selects a rectangular subimage from the original image. We propose average-case metrics for measuring the resilience of embedding patterns in the presence of cropping attacks based on the idea of a minimal cropping region, and give the values of these metrics for various embedding patterns.

\section{Related Work}

Our problem is similar to the problem of multi-dimensional data de-clustering, used in databases to reduce latency when retrieving data from multiple disks using parallel I/O. In this problem, the time required to process a query is proportional to the greatest amount of data retrieved from a single disk.

A range query is a query formed by taking all of the data points that lie between a given maximum and minimum value in each dimension, i.e. a kind of "cropping" of the data set. To minimise the time required to satisfy such a query, we want the segments of data in the range query to be distributed as homogeneously as possible over all disks. If we think of the disks as watermarks, the similarity between this problem and ours becomes apparent.

The two problems are not quite identical, however. In the de-clustering problem, we wish to minimise the number of segments in any region that lie on a single disk. In our waterkmarking problem, we wish to maximise the number of different watermarks in any region. Nonetheless, an allocation that is good for one seems likely to be a good choice for the other one.

Prabhakar, et al. [5] give a survey of de-clustering methods and propose a general paradigm (the cyclic paradigm) for de-clustering that generalises many previously-existing schemes. They compare them by computing the average cost of a query over all possible range queries. Obviously this approach becomes impractical for large data sets, and they later resort to using a randomly-chosen subset of all possible queries. In this paper, we propose a deterministic method of choosing the subset of cropping regions to be tested such that the set chosen gives a good representation of the performance of the embedding pattern against an arbitrary attack.

Atallah and Frikken [2] define an area as complete if it contains at least one copy of all watermarks (equivalently, uses all disks), and full if it does not contain any duplicate watermarks (or disks). They then define two metrics called the maximum non-complete area (MNCA) and minimum non-full area (MNFA). They show that the optimal values for the MNCA and MNFA can be achieved only for a number of watermarks $m=1,2,3$ or 5 , in the sense that an "optimal" pattern is one in which any rectangle of area at least $m$ contains all watermarks.

LNCS 2939 @ Springer-Verlag 
They furthermore show how to construct embedding patterns that have a nonoptimal MNCA of $O(m)$ and a non-optimal MNFA of $\Omega(m)$. They also give an experimental evaluation of both metrics for a number of different embedding patterns taken from work in database de-clustering.

The notion of a complete area seems more applicable to the watermarking problem than the notion of a full one, since in this case we are only interested in whether or not watermarks are present and not so much in whether or not they are duplicated (the latter is more of an issue in the data de-clusterig problem). The MNCA is a measure of the worst-case performance of an embedding pattern in the presence of cropping attacks. In this paper, we will introduce metrics that measure the average-case performance of embedding patterns in terms of the completeness of cropped regions.

\section{Embedding Patterns}

For the purposes of this study, an embedding pattern is a map $\phi: \mathcal{N} \times \mathcal{N} \rightarrow \mathcal{A}$ from a two-dimensional space to an $m$-element data set $\mathcal{A}=\{0, \ldots, m-1\}$, where $m$ is the number of watermarks to be embedded. The segment at position $(x, y)$ in the segmentation grid will receive watermark $\phi(x, y)$.

\subsection{Cyclic Methods}

Many of the methods currently used for data de-clustering can be categorised as cyclic methods [5]. In these methods, we set

$$
\phi(x, y)=(x H+y J) \bmod m
$$

for some integers $H$ and $J$. For example, the "disk modulo" method [3] is a cyclic method with $H=J=1$ and the "optimal" method [1] is a cyclic method with $H=\left\lfloor\frac{m}{2}\right\rfloor$ and $J=1$. The latter method is optimal for a few special values of $m$ in the sense that no range query of size $T$ requires more than $\left\lceil\frac{T}{m}\right\rceil$ disks.

If we choose $H$ and $J$ to be relatively prime to $m$, we will guarantee that every element of $\mathcal{A}$ will appear in the pattern (assuming that the range of $x$ and $y$ is large enough). In this paper, we will always choose $J=1$ but allow $H$ to vary, as in [5].

\subsection{Fieldwise Exclusive Method}

The fieldwise exclusive (FX) method [4] was introduced for the case when $m$ is a power of two, however, its definition is also valid for arbitrary $m$. In this method, we set

$$
\phi(x, y)=x \oplus y \bmod m^{\natural},
$$

where ' $\oplus$ ' denotes the bit-wise exclusive-OR function.

LNCS 2939 @ Springer-Verlag 


\subsection{Tile Rotation Method}

It is possible for an embedding pattern to have an unbounded MNCA if it contains incomplete rows or columns, that is, an $a \in \mathcal{A}$ such that $\phi(x, y) \neq a$ for any $x$ with fixed $y$, or any $y$ with fixed $x$. To avoid this, we would like to ensure that every row and column of the pattern contains every watermark. We can achieve this by forming a large pattern from rotations of a basic complete pattern, which we will call the tile rotation method.

Let $m^{\prime}=a \times b$ be the smallest composite number at least as large as $m$. Consider an $a \times b$ tile $L$ consisting of all the elements of $\mathcal{A}$ in an arbitrary arrangement (note there will be empty positions if $m^{\prime}>m$ ). Let $\operatorname{rotr}(L, i)$ denote and $i$-column rotation of the rows of $L$, and $\operatorname{rotc}(L, i)$ denote rotation of the columns similarly. Then we can define an embedding pattern by dividing the segmentation grid into $a \times b$ blocks and assigning $\operatorname{rotr}(\operatorname{rotc}(L, j), i)$ to the block at $(i, j)$. That is, set

$$
\begin{aligned}
\phi(x, y) & =\operatorname{rotr}\left(\operatorname{rotc}\left(L,\left\lfloor\frac{y}{b}\right\rfloor\right),\left\lfloor\frac{x}{a}\right\rfloor\right)(x \bmod a, y \bmod b) \\
& =\operatorname{rotc}\left(L,\left\lfloor\frac{y}{b}\right\rfloor\right)\left(x+\left\lfloor\frac{x}{a}\right\rfloor \bmod a, y \bmod b\right) \\
& =L\left(x+\left\lfloor\frac{x}{a}\right\rfloor \bmod a, y+\left\lfloor\frac{y}{b}\right\rfloor \bmod b\right)
\end{aligned}
$$

\section{Measurement Methods}

For the purposes of watermark detection, a segment can be considered to have been completely eliminated if there is an insufficient part of it remaining to reliably detect the watermark after cropping, and to be wholly intact otherwise. Hence we can consider all cropping to take place along the segment boundaries.

In principle, an attacker may crop an image to some arbitrary rectilinear polygon. However, non-rectangular images are of limited value and, furthermore, could not reasonably be accepted as a proof of ownership since they have obviously been tampered with. Our metrics will consider only rectangular regions to be interesting.

In general, no rectangle of the original image is any more valuable to the attacker than any other. We therefore model a cropping attack as the random selection of a rectangular area of the segmentation grid.

\subsection{Minimal Cropping Regions}

Ideally, we would like every area of size $m$ or greater to contain the complete set of watermarks. However, Atallah and Frikken have shown that this is possible only for a very limited choice of $m$. One method of measuring the performance of an embedding pattern, then, might be to test all regions of size $m$, and count the number of attacks that would succeed, that is, count the number of points at which the embedding pattern fails to be optimal.

LNCS 2939 @ Springer-Verlag 
However, this can give misleading results if $m$ has few factors since there will not be many rectangles with area exactly $m$ and this selection of rectangles may not be very representative of the collection of possible cropping attacks. In particular, if $m$ is prime, only $1 \times m$ and $m \times 1$ rectangles will be tested, completely ignoring attacks that take square or near-square regions.

On the other hand, an obvious way of measuring the performance of an embedding pattern would be to test all possible cropped regions, and count the number of attacks that succeed, similar to the experiments performed in [5]. However, this leads to testing many very large areas that will never fail under any reasonable embedding pattern, and very small areas that cannot possibly contain all watermarks.

We define a minimal cropping region for area $T$ to be an $a \times b$ sub-image $C$ of $B$ such that

- if $a \leq b$ and $a \leq \sqrt{T}$, then $b=\left\lceil\frac{T}{a}\right\rceil$; and

- if $a>b$ and $b \leq \sqrt{T}$, then $a=\left\lceil\frac{T}{b}\right\rceil$.

Intuitively, the set of all minimal cropping regions for an area $T$ is the set of all rectangles with area at least $T$ and minimal in each dimension. For example, the minimal cropping regions for $T=5$ are of size $5 \times 1,3 \times 2,2 \times 3$ and $1 \times 5$.

An embedding pattern $\phi$ is periodic if $\phi(x+\delta x, y+\delta y)=\phi(x, y)$ for some periods $\delta x$ and $\delta y$; all of the patterns considered in this paper are periodic. Our measurements consist of placing every possible minimal cropping region at every possible position over one period of the pattern, and counting the number of incomplete regions found. This reduces the number of tests required by a linear factor compared to testing all regions, since the number of minimal cropping regions is $O(m)$ but the number of all possible regions is $O(\delta x \delta y)$ and all of the embedding patterns used in this study have $\delta x, \delta y \geq m$.

\subsection{Proposed Metrics}

We propose two new average-case metrics based on the concept of a minimal cropping region, and two variations of Atallah and Frikken's worst-case metrics.

The All-Exclusion (AE) Metric. We define the all-exclusion metric as the number of complete minimal cropping regions, divided by the the total number of minimal cropping regions. This metric gives an indication of the general robustness of the embedding pattern.

The Single-Exclusion (SE) Metric. We define the single-exclusion metric as the probability that a minimal cropping region will contain any given watermark. Let $c_{n}$ be the proportion of minimal cropping regions that exclude $n$ watermarks. Then we can compute the SE metric as

$$
\mathrm{SE}=1-\sum_{n=1}^{m} \frac{c_{n} n}{m} \cdot
$$

LNCS 2939 @ Springer-Verlag 
This metric favours cropping regions that are closer to being complete (i.e. do not exclude many watermarks) and gives an indication of how evenly distributed the watermarks are.

The (modified) Maximum Non-Complete Area (MaxNCA). For ease of computation, we modify Attallah and Frikken's notion of the maximum non-complete area to incorporate minimal cropping regions. Our modified maximum noncomplete area is the maximum integer $N$ such that there exists an incomplete minimal cropping region of size $N$. This metric gives an indication of the largest cropping attack that might succeed. As noted in Section 3.3, this value is unbounded if the pattern contains incomplete rows or columns (which would extend indefinitely through all periods of the pattern). In our tests, our program aborted with an error if it found an incomplete minimal cropping region of some large size chosen to control the programme's running time.

The Minimum Complete Area (MinCA). Similarly, we define the minimum complete area as the minimum integer $N$ such that there exists a complete minimal cropping region of size $N$. This metric gives an indication of the smallest cropping attack that might fail.

\section{Results}

We computed the values of all of the metrics described in Section 4.2 for $2 \leq$ $m \leq 10$ for each of the embedding patterns described in Section 3 .

For the cyclic method, we performed two series of tests with different step sizes $H$. The first has $H=1$, i.e. it is the "disk modulo" method.

Since the pattern for $H=m-a$ is symmetric to the pattern for $H=a$, we do not need to consider $H$ 's greater than half $m$. If $H$ is not relatively prime to $m$, the pattern will not include all watermarks. Therefore we chose the second $H$ to be the greatest number that is relatively prime to $m$ but not greater than half $m$; this is equivalent to the "relatively prime HalfM" method of Prabakhar, et al. We conjectured that this would be the best choice for $H$ since it maximises the difference between two adjacent rows, therefore minimising the number of repetitions within a rectangle covering two or more rows. Indeed, Prabakhar et al. show results indicating that this method is superior to all of the previouslyexisting methods for database de-clustering.

Figures 2 and 3 show our all-exclusion and single-exclusion metrics, respectively.

Figures 4 and 5 show the maximum non-complete area and minimum complete area, respectively. Note that the FX method with $m=6,7,9$ and 10 has an unbounded MaxNCA.

\section{Discussion}

As we might have expected from the observations made in Section 2, our results are broadly similar to those obtained by Prabhakar, et al. for data de-clustering.

LNCS 2939 @ Springer-Verlag 
$\longrightarrow$ Cyclic, $H=1$

$\multimap$ Cyclic, $H=$ maximum

- - - - FX

$\Delta \cdots .$. Tile rotation

Fig. 1. Graph legend

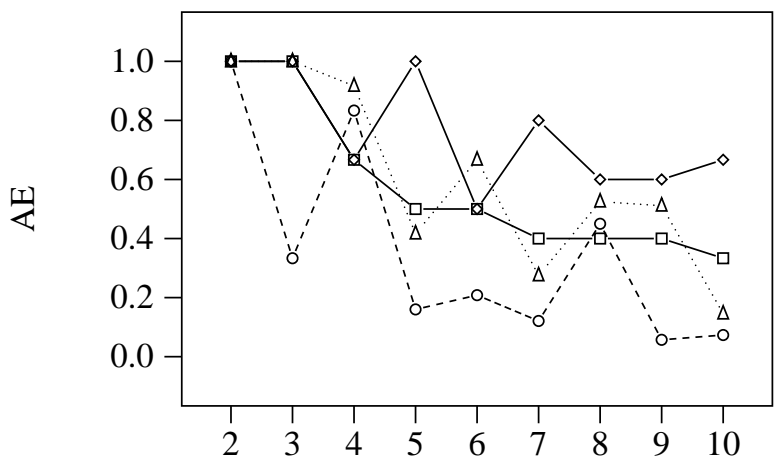

$m$

Fig. 2. All-exclusion metric

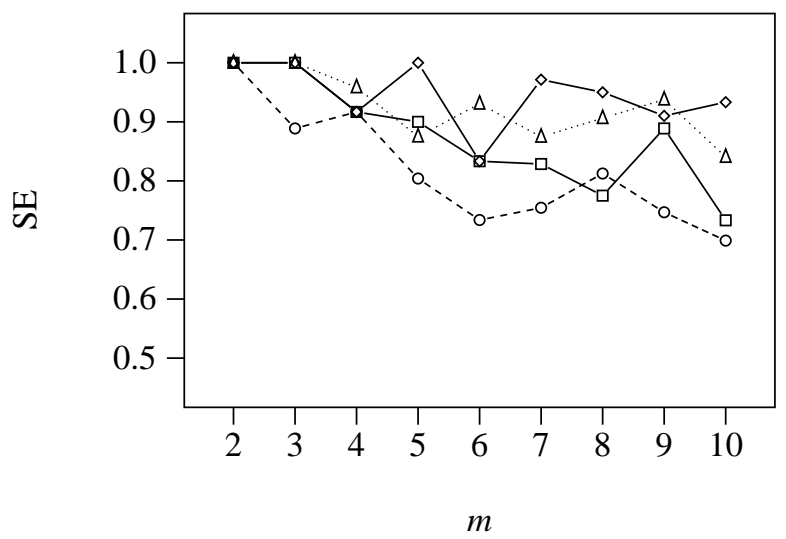

Fig. 3. Single-exclusion metric

LNCS 2939 (c) Springer-Verlag 


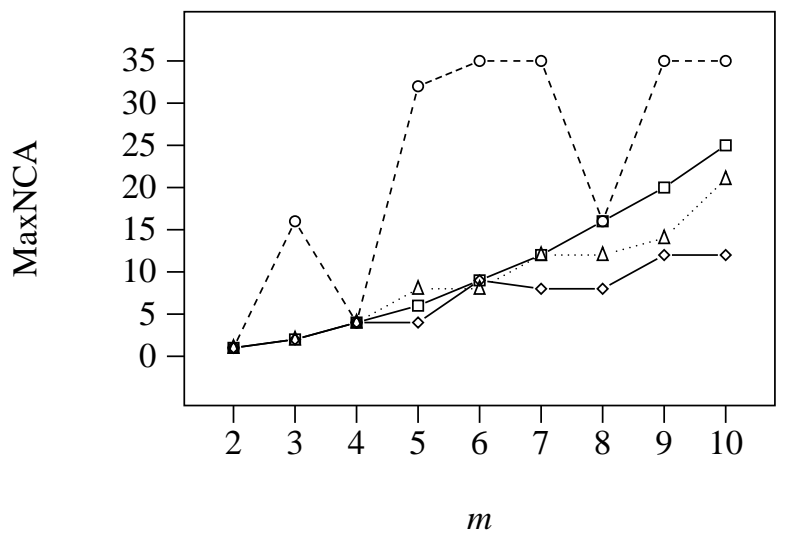

Fig. 4. (Modified) maximum non-complete area

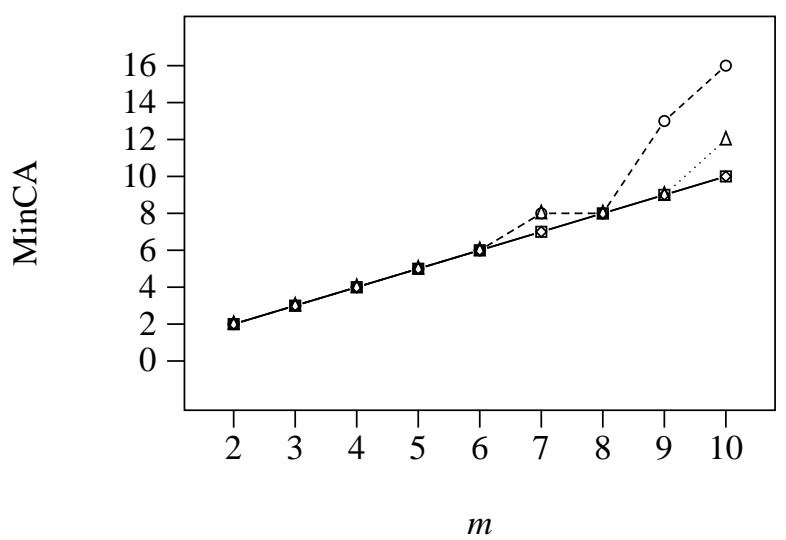

Fig. 5. Minimum complete area

LNCS 2939 (C) Springer-Verlag 
The cyclic method with maximum step generally scores the best results, and always improves on the cyclic method with minimum step. The FX method scores reasonably well when $m$ is a power of 2 , but poorly for other values of $m$.

The new tile rotation method generally scores a little worse than the maximumstep cyclic method, but scores better for $m=4$ and 6 . In these two cases, the highest relatively prime number less than half $m$ is just 1 , meaning that the maximum-step method is the same as the minimum-step method.

On the other hand, there are dips in the score for the tile rotation method at $m=5$ and 7 , which are prime numbers. This might be expected since the basic tile from which the pattern is constructed will have an empty position if $m$ is prime (recalling that the tile must have composite area at least as great as $m$ ). The presence of empty positions obviously reduces the effectiveness of the pattern.

\section{Conclusion}

We have introduced two new metrics for measuring the average-case performance of embedding patterns for segmented watermark, based on the notion of a minimal cropping region. Using minimal cropping regions substantially reduces the number of tests required for testing the average-case performance of embedding patterns as compared to the brute force approach.

As in related work, our measurements favour embedding patterns based on the cyclic paradigm, and, in particular, cyclic methods with a large step size.

\section{References}

1. K. Abdel-Ghaffar and A. El Abbadi. Optimal allocation of two-dimensional data. In International Conference on Database Theory, pages 409-418, Delphi, Greece, 1997.

2. M. Atallah and K. Frikken. Cropping-resilient segmented multiple watermarking. In Workshop on Algorithms and Discrete Structures, pages 231-242, 2003.

3. H. C. Du and J. S. Sobolewski. Disk allocation for cartesian product files on multipledisk systems. ACM Transactions on Database Systems, 7:82-101, 1982.

4. M. H. Kim and S. Pramanik. Optimal file distribution for partial match retrieval. In ACM International Conference on Management of Data, pages 173-182, Chicago, USA, 1988.

5. S. Prabhakar, K. Abdel-Ghaffar, D. Agrawal, and A. El Abbadi. Efficient retrieval of multi-dimensional datasets through parallel I/O. In ACM Symposium on Parallel Algorithms and Architectures, pages 78-87, 1998.

6. N. P. Sheppard, R. Safavi-Naini, and P. Ogunbona. On multiple watermarking. In Workshop on Security and Multimedia at ACM Multimedia, pages 3-6, Ottawa, Canada, 2001. 